\title{
Stochastic Process for the Availability Assessment of Single-Feeder Industrial Energy System Sections
}

\author{
Tom Van Acker, Graduate Student Member, IEEE, and Dirk Van Hertem, Senior Member, IEEE
}

\begin{abstract}
Given the high interruption costs of industrial processes, dependent on the cause, duration and time, accurate availability assessment of the industrial energy systems supplying those processes is crucial in order to avoid unnecessary investments or indicate necessary improvements. A method is presented to sectionalize an industrial energy system based on its radial topology and recoverability options. Additionally, this paper introduces a novel non-renewal stochastic process able to compute the (un)availability of single-feeder sections with respect to cause, duration and time, taking into account competing risks, general transition rates and both minimal and perfect corrective maintenance. An accompanying solution strategy is developed which allows decoupling of the very diverse time constants. Finally, a numerical illustration is provided validating the proposed approach against the Markov and semi-Markov processes under their respective assumptions and illustrating the possible errors when modeling the problem using the aforementioned stochastic processes.
\end{abstract}

Index Terms - Industrial plants, availability, reliability, maintenance, stochastic process.

Sets

\section{NOMENCLATURE}

$\begin{array}{cl}\mathcal{A} & \text { Set of cohort variables } \\ \mathcal{C} & \text { Set of cycles } \\ \mathcal{F} & \text { Set of failures } \\ \mathcal{N} & \text { Singular subset of the normal operation state } \\ \mathcal{P} & \text { Subset of post-failure states } \\ \mathcal{R} & \text { Set of reduced cycles }(f, c) \\ \mathcal{S} & \text { Set of states }(=\mathcal{N} \cup \mathcal{P}) \\ \mathcal{T} & \text { Set of transitions } \\ \text { States } & \end{array}$

$\left(i, \varphi_{i}\right) \quad$ State description, where $i$ represents the state itself and $\varphi_{i}$ represents its sojourn time

$R_{i}\left(\varphi_{i}\right) \quad$ Survival function of a state $i$

$p_{i}\left(t, \varphi_{i}\right) \quad$ Probability of occupying a state $i$ at time $t$

$p_{p}\left(t, \varphi_{p}, \varphi_{f}\right)$ Extended probability of occupying a postfailure state $p$, where $\varphi_{f}$ represents the operation time at which the component failed

\section{Transitions}

$i j$

$f_{i j}\left(\varphi_{i}\right)$

$\rho_{i j}\left(\varphi_{i}\right)$

$\lambda_{n j}\left(\varphi_{n}\right)$

Transition description, representing a transition from state $i$ to $j$ pdf describing a transition $i j$ Transition rate describing a transition $i j$ Failure rate describing a transition from $n$ to a post-failure state $j$

T. Van Acker and D. Van Hertem are with the Departement of Electrical Engineering, KU Leuven, Leuven, Belgium (e-mail: tom.vanacker@esat.kuleuven.be; dirk.vanhertem@esat.kuleuven.be).

This research is funded by a Ph.D. Fellowship of the Flemish Agency for Innovation \& Entrepreneurship in cooperation with BASF Antwerp NV (Grant No. 150257).

The singular and plural of an acronym are always abbreviated the same.

\section{INTRODUCTION}

\section{A. Background and Motivation}

A $\mathrm{N}$ industrial energy system (IES) is designed to supply energy services to processes within an industrial site. Adequate energy service entails supplying energy with sufficient quality. The energy supply is of sufficient quality when the quality limits prescribed by the industrial processes are satisfied. Different energy vectors have different indicators quantifying quality. Electrical quality indicators include voltage, current and frequency, whereas quality indicators of flow systems include pressure and flow rate. If the IES state does not cause a violation of the quality limits, as prescribed by a certain industrial process, then the IES is said to be available for that industrial process, i.e., able to perform as required [1]. On the other hand, the IES becomes unavailable for a certain industrial process when any of the prescribed limits of that process are violated by the IES state, leading to an interruption of that process. Several surveys, including [2], [3], have shown that the financial losses due to interruption of industrial consumers significantly exceed those of other consumers, e.g., residential or commercial. Furthermore, a significant discrepancy exists between the interruption costs of different industrial processes within an industrial site. Additionally, these surveys have shown that the financial losses largely depend on three characteristics describing the quality limit violation that causes the industrial process interruption: 1) cause, which quality limit was violated; 2) duration, how long was the quality limit violated; and 3) time, when was the quality limit violated. Two real-world examples in the field of industrial power systems make this more clear. First, a failure in the system may result in a short-circuit, which in turn causes a voltage dip throughout the network. Specific electrical equipment have different voltage dip withstand capabilities both in terms of duration and voltage magnitude experienced by the device. The latter depends on the location of the short circuit, or more specifically the cause of the quality limit violation. A complete discussion on the topic can be found in [4]. Second, fault clearing in a radial network isolates a part of the system, leaving some industrial processes without power. In case of aluminum smelters [5], the cost associated with loss of supply is initially limited, whereas a power interruption of a couple of hours may result in cracking of the melting pot, significantly reducing cell life. Even further cooling results in aluminum solidification, amounting to even higher costs.

An IES operator should balance the cost of quality against the value of quality when designing, maintaining and operating its energy system. This entails minimizing the sum of its 
utility cost and unavailability risk. The utility cost is the cost of designing, maintaining and operating the IES. The unavailability risk is the product of the probability and severity of the unavailability; the latter is usually expressed as the cost representative of the financial losses due to the unavailability. Inaccurate assessment of the unavailability results in an overor underestimation of the unavailability risk, which in turn leads to over- or underinvestment in the IES, resulting in a total system cost which is not minimal. This paper presents a non-renewal stochastic process that allows for a more accurate availability assessment of a single-feeder IES section.

\section{B. Contributions and Organization of the Paper}

This paper presents an approach to sectionalize an IES based on its radial topology and build-in recoverability options, allowing for a more in-depth availability assessment whilst keeping the problem tractable. Additionally, a novel stochastic process and an accompanied solution strategy are presented, able to determine the state probabilities for single-feeder IES sections. Following features are included, enabling more accurate availability assessment:

- State probabilities in function of time and state sojourn time, allowing to distinguish between cause, duration and time of the unavailability.

- General time-homogeneous transition rates in function of the current state sojourn time, allowing for a more accurate representation of the availability characteristics.

- Incorporation of competing risks as well as perfect and minimal maintenance, allowing for a more realistic representation of the availability characteristics.

- A solution strategy which decouples larger from the smaller time constants, allowing for more appropriate step-sizes.

The paper is organized as follows: section II summarizes availability terminology and reviews the relevant stochastic processes. Section III discusses the general IES structure and presents an appropriate sectionalizing approach. Section IV defines the mathematical framework describing the availability of a single-feeder IES section and the dynamical behavior of the state probabilities. Section $\mathrm{V}$ discusses a solution strategy which allows determining the section (un)availability. Section VI presents a numerical illustration validating the presented stochastic process against the Markov and semiMarkov methods under their respective assumptions. Finally, section VII concludes the paper.

\section{Availability and Stochastic Processes}

\section{A. Availability, Reliability, Maintainability and Recoverability}

The IES availability with respect to a certain industrial process is an attribute of the IES state and depends on the combined characteristics of its reliability, maintainability, and recoverability, each describing possible state transitions. Each of these availability characteristics has highly diverse time constants ranging from $100 \mathrm{~ms}$ to several decades (Fig. 1). Reliability is defined as the ability to perform as required, without failure, for a given time interval, under given conditions [6]. Any IES component can fail in several ways, each

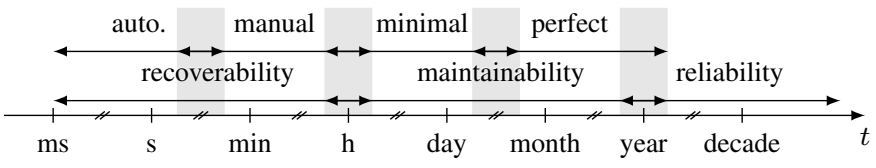

Fig. 1. Time constants governing the availability characteristics' transitions.

corresponding to their individual failure rate and subsequent maintainability and recoverability actions, i.e., competing risks. Maintainability is defined as the ability to be retained in, or restored to, a state to perform as required, under given conditions of use and maintenance [7]. Retaining the system in an available state is referred to as preventive maintainability, whereas restoring the system to an available state following a failure is referred to as corrective maintainability. For the purpose of this paper, only the latter is considered. Maintainability is generally categorized based on its restoration degree [8]: 1) perfect, restored to a state as-good-as-new; 2) minimal, restored to a state as-bad-as-old; 3) imperfect, restored to a state in between as-good-as-new and as-bad-as-old; and 4) worse, restored to a state worse-than-old. Only the first two types of maintenance are considered in this paper. Recoverability is defined as the ability to recover from a failure, without corrective maintenance [9]. This encompasses all actions, both automatic and manual, which restore the availability of (part of) the IES, excluding maintenance actions, e.g., fault clearing actions, restorative topological actions and activation of backup energy supply.

\section{B. Stochastic Processes Review}

The stochastic nature of IES availability can be modeled using a state-transition diagram with a discrete state-space and continuous time-space. This subsection reviews stochastic processes able to compute the state probabilities necessary to determine the unavailability risk, each with their respective assumptions and consequent reflections on their accuracy.

A well-known stochastic process is the Markov process, which derives its name from the fact that it respects the Markov or memoryless property. In a continuous time-space, the Markov property entails that all transition rates are constant, i.e., have an underlying exponential probability density function (pdf) with respect to the current state sojourn time [10]. The state sojourn time is defined as the amount of time spent in a state before leaving that state. Many authors have considered modeling system availability assessment using a Markov process, examples include [11]-[13]. However, using a Markov process has several drawbacks. First, it is not straightforward to express the state probability in function of both time and sojourn time when the process is not in steady state. Additionally, several papers have shown that using a Markov process, and consequently reducing the model to meet the Markov property, may introduce significant errors in the availability assessment. Bowles [14] has shown that a failure rate under the Markov property can greatly understate the system reliability for some periods and overstate it for others, which in turn introduces significant errors in the availability assessment. This is especially the case when 
taking competing risks into account, even for failures with an underlying exponential pdf [15]. Furthermore, Edimu et al. [16] show that assuming a constant maintainability or recovery rate also induces significant errors, as such actions generally are described by more densely concentrated pdf with shorter tails compared to their exponential counterparts. A more accurate approach should allow for general transition pdf and corresponding rates, enabling a more accurate description of the transitions.

A generalization of the Markov process, named the semiMarkov process, allows for such general transition rates by introducing a waiting time following arrival in a certain state [17]. The transitions themselves are still Markovian as they only depend on the current state, however the waiting time in a certain state is not necessarily Markovian, hence its name: semi-Markov process. Within the semi-Markov framework, it is possible to express state probabilities in function of both time and sojourn time using the Devooght-approach as presented in [18]. Many authors have considered modeling system availability assessment using a semi-Markov processes, examples include: [19], [20]. A semi-Markov process, similar to the Markov process, is a renewal process, which entails that it probabilistically starts over at each arrival epoch [21]. This means that a state is entered with a zero sojourn time following a transition, and consequently only perfect maintenance can be directly considered. A possible way to include other maintenance strategies is to include degradation states for the IES components [22]. The inclusion of these degradation states enables modeling minimal maintenance as re-entering the previously occupied degradation state. However, this approach has two distinct disadvantages. Firstly, under the assumption of continuous degradation, resetting the sojourn time induces a considerable error with respect to the overall component operation time, which can only be reduced by increasing the number of degradation states. Secondly, this method introduce additional states, reducing computational efficiency, as this is related to the number of states [18].

An additional drawback of both methods is that their numerical evaluation requires using a step-size related to the smallest state time constant, although such small step-sizes are often unnecessary to accurately evaluate other states. Including states with smaller time constants significantly decreases the computational efficiency of both the Markov and semi-Markov approach.

\section{CONCEPTUAL FRAMEWORK}

An IES is a complex system composed of a large number of components enabling on-site distribution of energy with the aim of supplying energy services to industrial processes. A typical IES topology is shown in Fig. 2. Sources can include on-site generation and utility connections. The IES topology differs considerably from public systems, e.g., distribution and transmission systems. Its basic structure is radial, i.e., a system consisting of single feeders supplied from a single source [23]. However, a set of backup feeders can be included to restore supply in case of feeder failure, depicted in grey in Fig. 2. Generally, these backup feeders are operated normally-open.

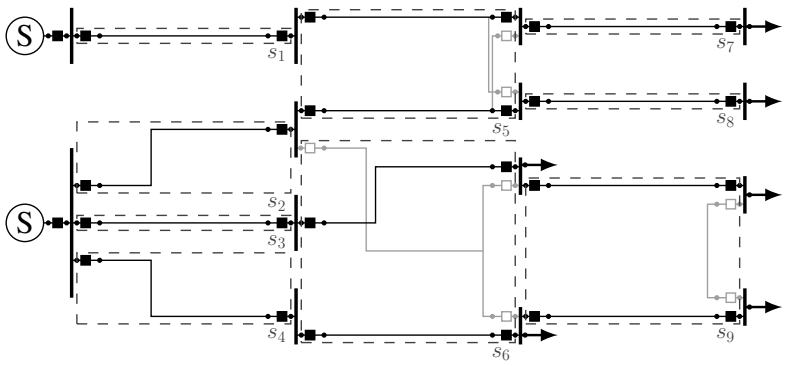

Fig. 2. Typical topology of an IES, where: node $(\bullet)$, feeder $(\bullet \bullet \bullet)$, source ( $(\mathrm{S}-\bullet)$, open/closed interrupter $(\bullet \square \bullet / \bullet \bullet \bullet)$, industrial process

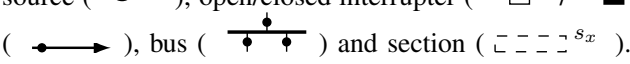

If they are operated normally-closed, the system is no longer radial.

Given this radial structure, protection of the feeder at its sending end is obligatory in order to isolate a possible fault from the source. Additionally, protection is required at the receiving end in case a fault can be fed by backup generation or via backup feeders. Following a failure of a certain component, all components within the same protection zone are isolated from the IES, exposing a clear dependency between these components. An additional dependency is introduced by the backup feeders. Based on these dependencies, an IES section is defined as a collection of IES components which have a direct dependency due to recoverability actions following any failure of one of the included components. Depending on the type of dependency, a distinction is made between single-feeder and multi-feeder sections, respectively $s_{1}-s_{4}, s_{7}-s_{8}$, and $s_{5}$ $s_{6}, s_{9}$ in Fig. 2. A single-feeder section consists of a set of series connected components, which have a negative failure dependency due to the fact that they are protected by the same interrupters. A multi-feeder section consists of multiple feeders in parallel, which can provide redundancy for other section feeders, introducing a positive failure dependency. Each of these feeders of a multi-feeder section coheres to the singlefeeder section structure.

The stochastic behavior of a dynamic system can be modeled by a state-transition diagram, likewise for an IES section. Four operation state types of an IES section are defined:

Normal State where all normally-closed feeder components are in operation;

Fault State during a fault following an IES component failure;

Post-fault State following clearing of a fault;

Corrected State following (partial) restoration of supply.

The latter three types are referred to as post-failure states. These are (partial) unavailable IES section states, each resulting in different quality limit violations and consequent effects and costs towards the connected industrial processes. Changes in the system are represented by transitions between states. As defined in Section II-A, a distinction is made between three transition types and corresponding rates:

Failure $\quad$ Failure of a component $(\lambda)$;

Maintenance Execution of a maintenance action $(\mu)$;

Recovery Execution of a recovery action $(\xi)$. 


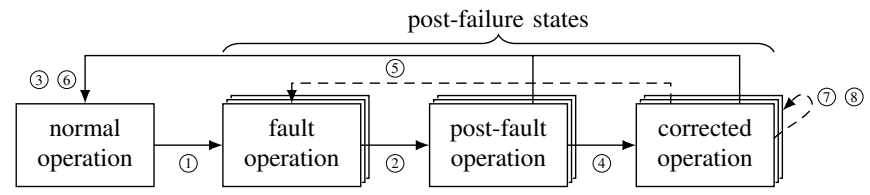

Fig. 3. Example of a state-transition diagram of an IES section. The dashed transitions are only possible in case of multi-feeder sections.

Fig. 3 depicts the state-transition diagram resulting from the aforementioned states and transitions. In the normal operation state, all IES section components of the normally-closed feeders are prone to failure. A failure results in a fault, impacting the system behavior. This entails a transition (1) to the fault operation state, which is governed by the failure rate $\lambda$. A fault is generally cleared following isolation of the faulty feeder by opening its corresponding interrupters. Such recoverability actions entail a transition (2) to a post-failure operation state and are governed by a recoverability rate $\xi$. It is assumed that no additional failures can occur during any fault operation state. Two different transitions can occur from a post-failure operation state. A first transition (3) to the normal operation state entails the repair of the failed component and is governed by a maintenance rate $\mu$. A second transition (4) to a corrected post-fault operation state follows a recoverability action, e.g., topological actions, and is governed by a recoverability rate $\xi$. In such states, the availability of the section is (partially) restored compared to the preceding post-fault state. For singlefeeder sections, no additional failures can occur in a corrected operation state as all feeder components are isolated following fault clearing. This negative failure dependency is directly taken into account in the design of the stochastic process. This means that the transition (6) is the only possible transition from a corrected operation state for single-feeder section, entailing maintenance of the failed feeder component. In case of multi-feeder sections, four different transitions can occur from a corrected operation state. A first transition (5) being an additional failure with corresponding failure rate $\lambda$, repeating the previously described cycle. A second and third transition (6), (7) entail maintenance with corresponding maintenance rate $\mu$. The former entails a transition to the normal operation state, following maintenance of a single failed component. Whereas, the latter entails a transition to another corrected operation state, following the maintenance of one of multiple failed components of different feeders. The last transition (8) to another corrected operation follows a recoverability action and is governed by $\xi$. If a feeder provides redundancy for another feeder upon its failure, then the failure rate of the redundant feeder should reflect the additional stress on that feeder due to redundancy activation. Direct incorporation of this type of failure dependency in the presented stochastic process would introduce an age variable for each normally-closed and backup feeder, increasing the problem dimension and consequently its computational complexity. This paper focusses on a singlefeeder sections.

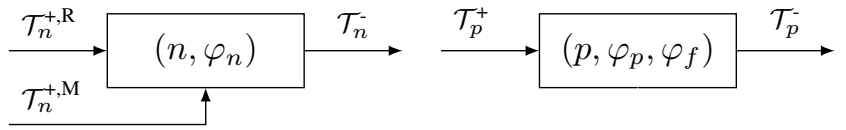

(a) Normal operation state

(b) Post-failure operation state

Fig. 4. Graphical representation of the two state types out of which a section state-transition diagram $(\mathcal{S}, \mathcal{T})$ is build up.

\section{Mathematical Framework}

The state transition diagram describing the considered stochastic process is modeled as a directed graph $(\mathcal{S}, \mathcal{T})$ with a state space $\mathcal{S}$ which includes states $\left(i, \varphi_{i}\right)$, and a transition set $\mathcal{T}$ which describes transitions $i j$ between states $\left(i, \varphi_{i}\right),\left(j, \varphi_{j}\right) \in \mathcal{S}$. Each state $\left(i, \varphi_{i}\right) \in \mathcal{S}$ is discrete with respect to the state $i$ itself but continuous with respect to its sojourn time $\varphi_{i}$. The state sojourn time $\varphi_{i}$ is the amount of time spent in a state $i$ before leaving that state. The probability of the stochastic process occupying a state $i$ for a certain sojourn time $\varphi_{i}$ at a time $t$ is defined as $p_{i}\left(t, \varphi_{i}\right)$, with $t, \varphi_{i} \in \mathbb{R}_{+}$and $p_{i}\left(t, \varphi_{i}\right) \in[0,1]$. The state space $\mathcal{S}$ is composed of one normal operation state $\left(n, \varphi_{n}\right) \in \mathcal{N}$ and multiple post-failure states $\left(p, \varphi_{p}\right) \in \mathcal{P}$. A set $\mathcal{C}$ is defined for the directed graph $(\mathcal{S}, \mathcal{T})$ that contains all simple cycles $c$ consisting of the normal operation state $n$ as well as a subset of post-failure states $p$. A simple cycle is a closed sequence of states within a directed graph with no repetition of states or transitions, other than the repetition of the starting state. In the remainder of the paper, the term cycle is used when referring to a simple cycle.

Each transition $i j$ is described by a weighted pdf $f_{i j}\left(\varphi_{i}\right)$, depending on the current state sojourn time $\varphi_{i}$. For each state $i$, the following applies

$$
\sum_{i j \in \mathcal{T}} \int_{0}^{\infty} f_{i j}\left(\varphi_{i}\right) \mathrm{d} \varphi_{i} \leq 1, \quad \forall\left(i, \varphi_{i}\right) \in \mathcal{S}
$$

where any state $i$ with an aggregate strictly less than one is referred to as a trapping state. The survival function $R_{i}\left(\varphi_{i}\right)$ for a state $i$ is equal to

$$
R_{i}\left(\varphi_{i}\right)=1-\sum_{i j \in \mathcal{T}} \int_{0}^{\varphi_{i}} f_{i j}\left(\varphi_{x}\right) \mathrm{d} \varphi_{x}, \quad \forall\left(i, \varphi_{i}\right) \in \mathcal{S} .
$$

For each transition $i j$, a transition rate $\rho_{i j}\left(\varphi_{i}\right)$ can be defined as

$$
\rho_{i j}\left(\varphi_{i}\right)=f_{i j}\left(\varphi_{i}\right) / R_{i}\left(\varphi_{i}\right), \quad \forall i j \in \mathcal{T}
$$

The normal operation state $\left(n, \varphi_{n}\right)$ describes the state where all normally-closed feeder components are in operation, and consequently the section is able to perform as required (Fig. 4a). The sojourn time $\varphi_{n}$ represents the operation time of the section components. In the general case, each component has its own operation time, changing $\left(n, \varphi_{n}\right)$ into $\left(n, \bar{\varphi}_{n}\right)$. However, this generalization is not considered in this paper, and all components are assumed to have a uniform $\varphi_{n}$. A transition away from the normal operation state $n$ is only possible when one of the section components experiences a failure, resulting in a transition $n j \in \mathcal{T}_{n}^{-}$to a post-failure 
state $j$. The associated transition rate is referred to as the failure rate $\lambda_{n j}\left(\varphi_{n}\right)$ and depends on the normal operation state sojourn time $\varphi_{n}$. A transition to the normal operation state $\left(n, \varphi_{n}\right)$ occurs when the section has successfully undergone maintenance. As both minimal and perfect maintenance are considered, two types of transitions to the normal operation state must be included (Fig. 4a). A first type: in $\in \mathcal{T}_{n}^{+, \mathrm{M}}$, describing minimal corrective maintenance, enters the normal operation state with a sojourn time $\varphi_{n}$ equal to the operation time $\varphi_{f}$ at which the component previously failed, i.e., the operation time of the failed component is retained. Whereas the second type: in $\in \mathcal{T}_{n}^{+, \mathrm{R}}$, describing perfect corrective maintenance, enters the normal operation state with a sojourn time $\varphi_{n}$ equal to zero, i.e., the failed component is replaced by a new one. If components are governed by a uniform $\varphi_{n}$, all related components are replaced upon the replacement of any failed component requiring perfect maintenance.

A post-failure state $\left(p, \varphi_{p}\right)$ describes any state which is not the normal operation state $\left(n, \varphi_{n}\right)$ (Fig. $4 \mathrm{~b}$ ). When the section is in a post-failure state, it is possible that the section is (partially) unable to perform as required due to quality limit violations, which can result in an industrial process interruption. In order to incorporate minimal repair, where the operation time of the failed component is retained, the operation time $\varphi_{f}$ at which the component failed needs to be passed along to the subsequent post-failure states, extending the post-failure state definition and associated probability respectively to $\left(p, \varphi_{p}, \varphi_{f}\right)$ and $p_{p}\left(t, \varphi_{p}, \varphi_{f}\right)$. The overall postfailure state probability $p_{p}\left(t, \varphi_{p}\right)$ can be found using

$$
p_{p}\left(t, \varphi_{p}\right)=\int_{0}^{\infty} p_{p}\left(t, \varphi_{p}, \varphi_{f}\right) \mathrm{d} \varphi_{f} .
$$

A transition $p j \in \mathcal{T}_{p}^{-}$away from a post-failure state $p$ to any state $j$ is only possible when the considered recoverability or corrective maintenance action has successfully been completed. A transition $i p \in \mathcal{T}_{p}^{+}$to a post-failure state $\left(p, \varphi_{p}, \varphi_{f}\right)$ comes either from the normal operation state or from another post-failure state. A post-failure state is always entered at a sojourn time $\varphi_{p}$ equal to zero.

The dynamic behavior of the normal state probability $p_{n}\left(t, \varphi_{n}\right)$ is described by (5)-(6):

$$
\begin{aligned}
\frac{\partial p_{n}}{\partial t}+\frac{\partial p_{n}}{\partial \varphi_{n}}= & -\sum_{n j \in \mathcal{T}_{n}^{-}} \lambda_{n j}\left(\varphi_{n}\right) p_{n}\left(t, \varphi_{n}\right) \\
& +\sum_{i n \in \mathcal{T}_{n}^{+, \mathrm{M}}} \int_{0}^{\infty} \rho_{i n}\left(\varphi_{i}\right) p_{i}\left(t, \varphi_{i}, \varphi_{n}\right) \mathrm{d} \varphi_{i}, \\
p_{n}(t, 0)= & \sum_{i n \in \mathcal{T}_{n}^{+, \mathrm{R}}} \int_{0}^{\infty} \int_{0}^{\infty} \rho_{i n}\left(\varphi_{i}\right) p_{i}\left(t, \varphi_{i}, \varphi_{f}\right) \mathrm{d} \varphi_{i} \mathrm{~d} \varphi_{f} .
\end{aligned}
$$

Whereas, the dynamic behavior of the state probabilities $p_{p}\left(t, \varphi_{p}, \varphi_{f}\right)$ for each post-failure state $p$ is described by (7)-(8):

$$
\begin{aligned}
\frac{\partial p_{p}}{\partial t}+\frac{\partial p_{p}}{\partial \varphi_{p}}= & -\sum_{p j \in \mathcal{T}_{\bar{p}}^{-}} \rho_{p j}\left(\varphi_{p}\right) p_{p}\left(t, \varphi_{p}, \varphi_{f}\right), \\
p_{p}\left(t, 0, \varphi_{f}\right)= & \sum_{i p \in \mathcal{T}_{p}^{+}: i \in \mathcal{N}} \lambda_{i p}\left(\varphi_{f}\right) p_{n}\left(t, \varphi_{f}\right) \\
& +\sum_{i p \in \mathcal{T}_{p}^{+}: i \in \mathcal{P}} \int_{0}^{\infty} \rho_{i p}\left(\varphi_{i}\right) p_{p}\left(t, \varphi_{i}, \varphi_{f}\right) \mathrm{d} \varphi_{i} .
\end{aligned}
$$

\section{Solution Strategy}

This section presents a solution strategy for the considered problem which allows determining the state probabilities and decouples the highly diverse time constants of the transitions. The strategy is based on the knowledge that for any determined set of equations, once one unknown is obtained, all other unknowns can be derived. Following this reasoning, the choice is made to obtain the normal operation state probability $p_{n}\left(t, \varphi_{n}\right)$, which subsequently allows deriving all post-failure state probabilities $p_{p}\left(t, \varphi_{p}\right)$. To this end, all postfailure state probabilities are expressed in function of the normal operation state probability and integrated into (5) and (6), after which the resulting equations can be solved using a numerical approach. The developed solution strategy is valid under the following conditions:

- The initial state of the process is the normal operation state $\left(n, \varphi_{n}\right)$, where $\varphi_{n} \in\left[0, \varphi_{n}^{\max }\right] \subset \mathbb{R}_{+}$.

- The state transition diagram $(\mathcal{S}, \mathcal{T})$ does not contain any other cycles other than those in $\mathcal{C}$, which all include the normal operation state $\left(n, \varphi_{n}\right)$.

- All components of the section have a uniform operation time $\varphi_{n}$.

Any cycle $c$ of the state-transition diagram $(\mathcal{S}, \mathcal{T})$ contains the normal operation state $\left(n, \varphi_{n}\right)$ and a subset of postfailure state $\left(p, \varphi_{p}, \varphi_{f}\right) \in \mathcal{P}_{c}$. For each cycle $c$, merging these post-failure states into a single state $\left(m, \varphi_{m}, \varphi_{f}\right)$ with a probability $p_{m}\left(t, \varphi_{m}, \varphi_{f}\right)$ reduces the cycle $c$ to a two-state cycle $(f, c) \in \mathcal{R}$ (Fig 5). Such a two-state cycle has two transitions, a first going from the normal operation state $n$ to the merged state $m$ and a second transition from the merged state $m$ to the normal operation state $n$. The combination of these two transitions, i.e., the reduced cycle, is uniquely described by $(f, c)$. The former transition is governed by the failure rate $\lambda_{f}\left(\varphi_{n}\right)$, i.e., the failure rate $\lambda_{n j}\left(\varphi_{n}\right)$, where $j$ is the first post-failure state of the cycle $c$. The latter transition is governed by a pdf $f_{c}\left(\varphi_{m}\right)$, which is the convolution of all transition pdf in between the merged post-failure states and the normal operation state of the cycle $c$, excluding the initial failure. Similar to any other transition, the pdf $f_{c}\left(\varphi_{m}\right)$

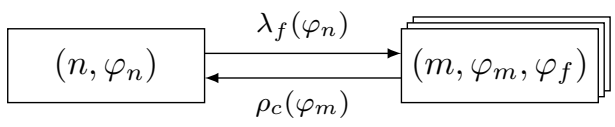

Fig. 5. Graphical representation of the state-transition diagram $(\mathcal{S}, \mathcal{T})$ if all cycles $c$ are reduced to two-state cycles $(f, c)$, i.e., all post-failure states of the original cycle $c$ are merged into one state $\left(m, \varphi_{m}, \varphi_{f}\right)$. 
has a corresponding transition rate $\rho_{c}\left(\varphi_{m}\right)$ using the survival function $R_{m}\left(\varphi_{m}\right)$. The dynamic behavior of $p_{m}\left(t, \varphi_{m}, \varphi_{f}\right)$ is governed by (7)-(8). Eq. (7) is a linear first-order partial differential equation, in mathematical biology often referred to as the McKendrick-von Foerster equation [24]. Such partial differential equations have the following analytical solution:

$$
p_{m}\left(t, \varphi_{m}, \varphi_{f}\right)=F\left(t-\varphi_{m}, \varphi_{m}, \varphi_{f}\right) R_{m}\left(\varphi_{m}\right),
$$

where $F\left(t-\varphi_{m}, \varphi_{m}, \varphi_{f}\right)$ represents the state boundary condition at a shifted point in time $t-\varphi_{m}$. Given (8), the state boundary condition can be formulated as:

$$
F\left(t-\varphi_{m}, \varphi_{m}, \varphi_{f}\right)=\lambda_{f}\left(\varphi_{n}\right) p_{m}\left(t-\varphi_{m}, \varphi_{f}\right),
$$

resulting in the following description for the state probability of the merged state:

$$
p_{m}\left(t, \varphi_{m}, \varphi_{f}\right)=\lambda_{f}\left(\varphi_{n}\right) p_{m}\left(t-\varphi_{m}, \varphi_{f}\right) R_{m}\left(\varphi_{m}\right) .
$$

Following reduction of all cycles $c \in \mathcal{C}$ into reduced cycles $(f, c)$ as presented in the previous paragraph, equations (5) and (6) can be reformulated into (12) and (13), where $\mathcal{R}^{\mathrm{M}}$ and $\mathcal{R}^{\mathrm{R}}$ represent those reduced cycles respectively requiring minimal and perfect maintenance:

$$
\begin{aligned}
\frac{\partial p_{n}}{\partial t}+\frac{\partial p_{n}}{\partial \varphi_{n}}= & -\sum_{(f, c) \in \mathcal{R}} \lambda_{f}\left(\varphi_{n}\right) p_{n}\left(t, \varphi_{n}\right) \\
& +\sum_{(f, c) \in \mathcal{R}^{\mathrm{M}}} \int_{0}^{\infty} \rho_{c}\left(\varphi_{m}\right) p_{m}\left(t, \varphi_{m}, \varphi_{n}\right) \mathrm{d} \varphi_{m}, \quad(12) \\
p_{n}(t, 0)= & \sum_{(f, c) \in \mathcal{R}^{\mathrm{R}}} \int_{0}^{\infty} \int_{0}^{\infty} \rho_{c}\left(\varphi_{m}\right) p_{m}\left(t, \varphi_{m}, \varphi_{f}\right) \mathrm{d} \varphi_{m} \mathrm{~d} \varphi_{f} .
\end{aligned}
$$

Combining (11), (12) and (13), the term $\rho_{c}\left(\varphi_{m}\right) R_{m}\left(\varphi_{m}\right)$ is replaced with $f_{c}\left(\varphi_{m}\right)$, following (3). By doing so, a convolution between $f_{c}\left(\varphi_{m}\right)$ and $p_{m}\left(t-\varphi_{m}, \varphi_{f}\right)$ becomes apparent, which can be rewritten by its shorthand $\left(f_{c} * p_{n}\right)\left(t, \varphi_{f}\right)$. The resulting (14) and (15) are a partial delay differential equation (PDDE) with a non-local boundary condition

$$
\begin{aligned}
\frac{\partial p_{n}}{\partial t}+\frac{\partial p_{n}}{\partial \varphi_{n}}= & -\sum_{(f, c) \in \mathcal{R}} \lambda_{f}\left(\varphi_{n}\right) p_{n}\left(t, \varphi_{n}\right) \\
& +\sum_{(f, c) \in \mathcal{R}^{\mathrm{M}}} \lambda_{f}\left(\varphi_{n}\right)\left(f_{c} * p_{n}\right)\left(t, \varphi_{n}\right), \\
p_{n}(t, 0)= & \sum_{(f, c) \in \mathcal{R}^{\mathrm{R}}} \int_{0}^{\infty} \lambda_{f}\left(\varphi_{f}\right)\left(f_{c} * p_{n}\right)\left(t, \varphi_{f}\right) \mathrm{d} \varphi_{f} .
\end{aligned}
$$

The literature on numerically solving PDDE with non-local boundary conditions is rather limited [25]. However, the problem structure permits to use the cohort strategy presented in [26] to discretize the solution space (Fig. 6). Each cohort variable $a \in \mathcal{A}$ links time $t$ and operation time $\varphi_{n}$, using:

$$
t=\varphi_{n}+a, \quad \forall a \in \mathcal{A} .
$$

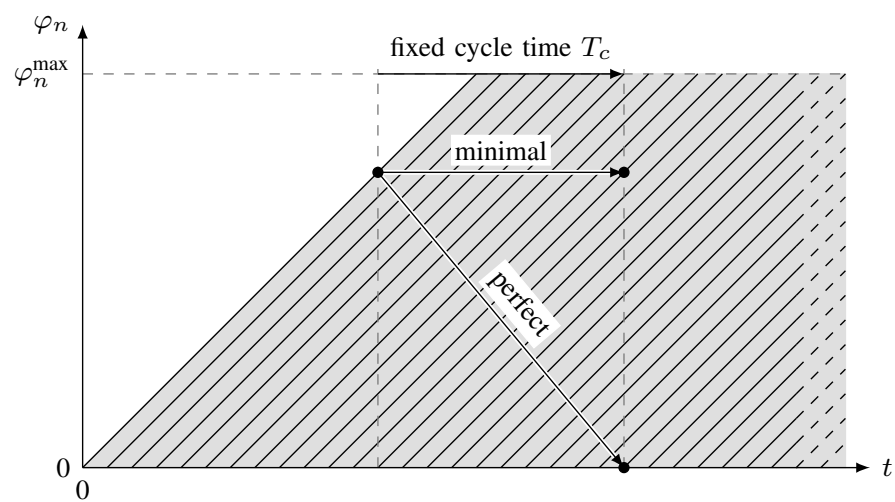

Fig. 6. Lexis diagram of the solution space. Under the assumption of $p_{n}(0,0)=1$, the grey area represents the solution space for the PDDE (14) and its boundary condition (15), where $\varphi_{n}$ is limited to $\varphi_{n}^{\max }$. The solution space for the set of ODE (17) and initial conditions (18) is represented by the black lines, one for each cohort variable $a$. Additionally, the two arrows represent the translations within the solution space representing minimal and perfect cycles for a fixed cycle time $T_{c}$.

This approach reduces the PDDE to a set of non-homogenous first order ordinary differential equations (ODE):

$$
\begin{aligned}
\frac{\mathrm{d} p_{n, a}}{\mathrm{~d} \varphi_{n}}= & -\sum_{(f, c) \in \mathcal{R}} \lambda_{f}\left(\varphi_{n}\right) p_{n, a}\left(\varphi_{n}\right) \\
& +\sum_{(f, c) \in \mathcal{R}^{\mathrm{M}}} \sum_{x \in X} f_{c, x} \lambda_{f}\left(\varphi_{n}\right) p_{a-x, n}\left(\varphi_{n}\right), \quad a \in \mathcal{A}, \\
p_{n, a}(0)= & \sum_{(f, c) \in \mathcal{R}^{\mathrm{R}}} \sum_{x \in X} \int_{0}^{\infty} f_{c, x} \lambda_{f}\left(\varphi_{f}\right) p_{a-x, n}\left(\varphi_{f}\right) \mathrm{d} \varphi_{f}, a \in \mathcal{A},
\end{aligned}
$$

where for a fixed cohort variable step-size $\mathrm{d} a$, the set $X=$ $\left\{1, \ldots,\left\lceil\varphi_{m}^{\max } / \mathrm{d} a\right\rceil\right\}$ and $f_{c, x}=\int_{(x-1) \mathrm{d} a}^{x \mathrm{~d} \alpha} f_{c}\left(\varphi_{m}\right) \mathrm{d} \varphi_{m}$. Numerical techniques for such equations are well-known in literature, both with regards to their stability as well as accuracy [27].

After finding a numerical solution for the normal operation state probability, it can be used to determine all post-failure state probabilities. Any post-failure $p$ can belong to multiple cycles $c$, each such combination is described by $f_{c, p}^{\mathrm{pre}}$ and $R_{c, p}$. For any cycle $c$, the former represents the convolution of all transitions in between the normal operation state $n$ and the considered post-failure state $p$, excluding the failure transition. The latter represents the survival function corresponding to the subsequent transition of the cycle $c$ away from the considered post-failure state $p$. Consequently, a post-failure state probability $p_{p}\left(t, \varphi_{p}\right)$ can be expressed as

$$
p_{p}\left(t, \varphi_{p}\right)=\sum_{(f, c) \in \mathcal{R}: c \in \mathcal{C}_{p}}\left(f_{c, p}^{\mathrm{pre}} * p_{f}\right)\left(t-\varphi_{p}\right) R_{c, p}\left(\varphi_{p}\right), \quad \forall p \in \mathcal{P}
$$

where $p_{f}(t)=\int_{0}^{\infty} \lambda_{f}\left(\varphi_{n}\right) p_{n}\left(t, \varphi_{n}\right) \mathrm{d} \varphi_{n}$.

The presented approach decouples the highly diverse time constants on two levels. First, the approach allows adjusting the time-step of a post-failure state to their respective time constant. Second, the normal operation probability of a cohort $a$ can be determined using a step-size relative to the time 
constant of the failures, while the spacing $\mathrm{d} a$ between these cohorts can be chosen relative to the minimal time constant of the cycles.

The Markov process computationally outperforms both the semi-Markov process and the presented approach, as it only requires the previous state probability to determine the next state probability, following the Markov property. Therefore, the Markov method is preferred whenever a problem satisfies the Markov property. Consequently, the following discussion only compares the semi-Markov method and the presented approach for non-Markovian problems. Both methods use a different approach to account for the non-Markovian nature of its transitions. The semi-Markov method solves a set of coupled integral equations, one for each state $\left(i, \varphi_{i}\right) \in \mathcal{S}$ [18]. On the other hand, the presented approach solves a convolution for each failure $f \in \mathcal{F}$ and cohort characteristic $a \in \mathcal{A}$. Depending on the ratio between $|\mathcal{S}|$ and $|\mathcal{F}|$, one method could outperform the other. However, as stated in section II-B, evaluating nonrenewal problems would require introducing additional states for the semi-Markov method, increasing $|\mathcal{S}|$, while $|\mathcal{F}|$ remains constant for the presented approach. Additionally, numerical evaluation of either the set of coupled integral equations or the convolution requires choosing an appropriate numerical step-size with respect to the chosen numerical method and the required accuracy. For the semi-Markov process, this stepsize $\mathrm{d} t$ should be chosen relative to the smallest time constant of its transitions. On the other hand, the step-size $\mathrm{d} a$ for the presented approach relates to the smallest time constant of the cycles. Depending on the problem instance, the step-size da may exceed the semi-Markov process step-size $\mathrm{d} t$.

\section{NUMERICAL ILLUSTRATION}

This section introduces a numerical illustration with the aim of validating the presented stochastic process and associated solution strategy. To this end, a test system is introduced which exhibits the features set forth in section I-B. Four test cases are introduced, each with different assumptions, to validate the proposed method against the Markov and semiMarkov methods under their respective assumptions and show the consecutive gain in modeling detail. The semi-Markov model is validated against the one presented in [28] and is subsequently used to validate the Markov model as the former generalizes the latter.

\section{A. Test System and Test Cases}

A small test section $s$ consisting of a single feeder is proposed (Fig. 7a). The only components prone to failure are those of section $s$. The section has two protection zones. Initially, a fault should be isolated by interrupters $i_{1}^{\mathrm{a}} / i_{1}^{\mathrm{b}}$; if unsuccessful, the fault is isolated by interrupters $i_{2}^{\mathrm{a}} / i_{2}^{\mathrm{b}}$. Whenever the fault is cleared by the second protection zone interrupters, the grid topology is manually altered to a state similar to where the fault was cleared by the first protection zone interrupters. Following the latter or following direct clearing of the fault by the first protection zone interrupters, the failed element is maintained and consequently restored to a working condition. The stochastic behavior of the test section is modeled by a

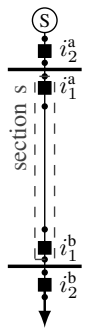

(a)

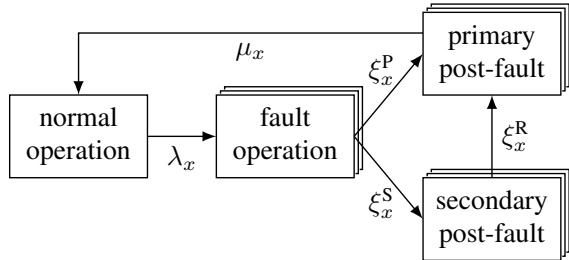

(b)
Fig. 7. Test section (7a) and associated state-transition diagram (7b). Each failure $x$ has its respective fault operation, primary and secondary post-fault states.

state-transition diagram (Fig. 7b) consisting of four types of states. Each post-failure state type has a separate state for each failure $x$. Table I gives an overview of the possible transitions between the different states.

Four test cases are introduced, each generalizing the previous test case, in order to showcase different results with respect to the test section availability. Each test case is defined by a three letter code. The first letter indicates the number of failures: S-single failure/M-multiple failures. The second letter indicates the underlying pdf type of the transitions: Eexponential/G-general. The third letter indicates the considered maintenance types: R-only perfect maintenance/B-both minimal and perfect maintenance.

1. $\mathbf{S} / \mathbf{E} / \mathbf{R}$ : The goal of the first test case is to validate the proposed approach against the Markov process. To this end, all underlying pdf of the transitions are exponential distributions (20), with $p$ and $a^{\chi}$ equal to one.

$$
f(\varphi)=p \frac{1}{\chi} \exp (-\varphi / \chi), \quad \varphi \geq 0 .
$$

2. $\mathbf{M} / \mathbf{E} / \mathbf{R}$ : The goal of the second test case is to illustrate the proposed approach's ability to exactly incorporate competing risks. To this end, three possible failures $x$ are included, respectively with a weighted overall probability $p_{x}$ of $0.3,0.4$ and 0.3 , and $a_{x}^{\chi}$ equal to $0.4,1.0$ and 2.5 .

3. $\mathbf{M} / \mathbf{G} / \mathbf{R}$ : The goal of the third test case is to validate the proposed approach against the semi-Markov process. To this end, the underlying pdf of transitions $\mu$ are modeled as Weibull distributions (21) and, $\xi$ are modeled as log-normal distributions (22).

$$
\begin{aligned}
& f(\varphi)=p \frac{\psi}{\chi}\left(\frac{\varphi}{\chi}\right)^{\psi-1} \exp \left(-(\varphi / \chi)^{\psi}\right), \quad \varphi \geq 0,(21) \\
& f(\varphi)=p \frac{1}{\varphi \psi \sqrt{(2 \pi)}} \exp \left(-\frac{\ln (\varphi)-\ln (\chi)}{2 \psi^{2}}\right), \quad \varphi \geq 0 .(22)
\end{aligned}
$$

TABLE I

TRANSITION TYPES FOR THE STATE-TRANSITION DIAGRAM (FIG. 7B)

\begin{tabular}{clccc} 
Symbol & Transition type & $p[-]$ & $\chi[\mathrm{h}]$ & $\psi[\mathrm{h}]$ \\
\hline$\lambda_{x}$ & Failure & $p_{x}$ & $a_{x}^{\chi} \cdot 10^{4}$ & - \\
$\xi_{x}^{\mathrm{P}}$ & Primary fault clearing & 0.8 & $10^{1}$ & 0.08 \\
$\xi_{x}^{\mathrm{S}}$ & Secondary fault clearing & 0.2 & $10^{1}$ & 0.08 \\
$\xi_{x}^{\mathrm{R}}$ & Topological action & 1.0 & $10^{2}$ & 0.08 \\
$\mu_{x}$ & Maintainability action & 1.0 & $10^{3}$ & 10
\end{tabular}




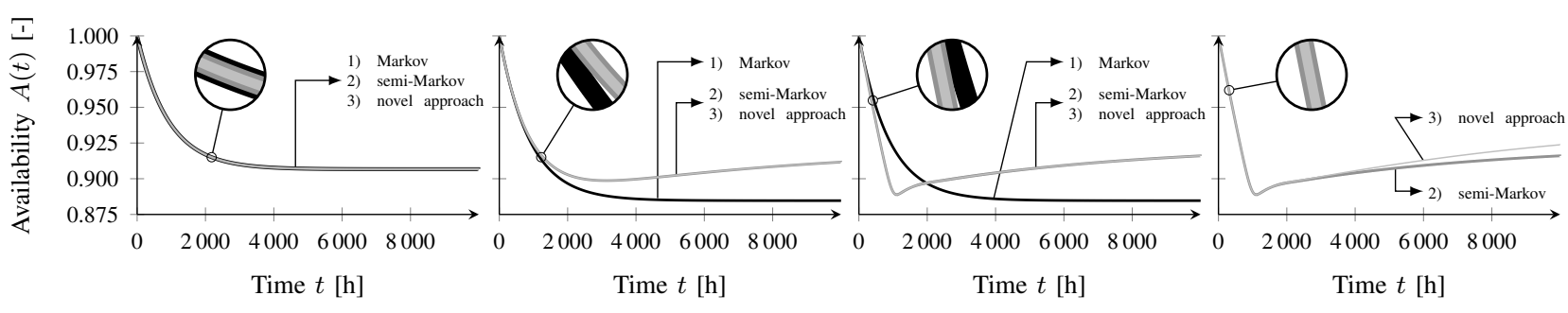

(a) Test case 1: (S/E/R)

(b) Test case 2: $(\mathrm{M} / \mathrm{E} / \mathrm{R})$

(c) Test case 3: (M/G/R)

(d) Test case 4: $(\mathrm{M} / \mathrm{G} / \mathrm{B})$

Fig. 8. Results for all test cases. Each test case is defined by a three letter code, where the first letter indicates the number of failures: S-single failure/M-multi failure. The second letter indicates the underlying pdf type of the transitions: E-exponential/G-general. The third letter indicates the considered maintenance type: R-only perfect maintenance/B-both minimal as perfect maintenance. Each test case is solved using: 1) Markov process ( - ), 2) semi-Markov process ( _ ) and 3) the novel approach proposed in this paper ( _ ) except for the last test case which is only solved using the latter two.

4. $\mathbf{M} / \mathbf{G} / \mathbf{B}$ : The goal of the last test case is to illustrate the ability to exactly incorporate minimal maintenance. To this end, the first two failures are followed by minimal maintenance, while the third failure still requires perfect maintenance.

\section{B. Results and Discussion}

Fig. 8 gives the availability with respect to time $t$ of the section for all four test cases. The only available state of the section $s$ with respect to the coupled industrial process is the normal operation state, all other states are unavailable states. Determining the availability with respect to time $t$ for the proposed approach requires

$$
A(t)=p_{n}(t)=\int_{0}^{\infty} p_{n}\left(t, \varphi_{n}\right) \mathrm{d} \varphi_{n}
$$

Fig. 8a shows the results of the first test case. All three methods return the same solution, benchmarking the proposed approach with the Markov process whenever the problem satisfies the Markov property. In the second test case, multiple failures are introduced with a different underlying exponential pdf. Fig. 8b shows a deviation between the results for the Markov process on the one hand and those for the semiMarkov and the novel approach on the other hand. As mentioned in [14], the Markov process is unable to exactly take competing risks into account, even if those transitions have an underlying exponential pdf. The second test case reiterates the conclusions made in the aforementioned paper. In the third test case, the underlying pdf of the maintenance and recovery transitions are respectively replaced by Weibull and log-normal distributions. Note that the results for the Markov process are still given for transitions with underlying exponential distributions, as the Markov process is unable to take general distributions into account. Fig. 8c gives an insight into the modeling error due to the invalid assumption of constant transition rates and shows the importance of being able to take general transition rates into account. Additionally, the third test case benchmarks the proposed approach against the semi-Markov process whenever the problem satisfies the renewal property. In the last test case, the problem no longer satisfies the renewal property as the maintenance following the first two failures is changed to minimal maintenance. Minimal maintenance retains the operation time following maintenance whereas perfect maintenance resets the operation time to zero. Whenever the failure rate is not constant, the choice of maintenance will have an effect on the availability. With respect to the system availability, minimal maintenance is preferred for decreasing failure rates, usually seen with infant failures. Perfect maintenance is preferred for increasing failure rates, usually seen with wear-out failures. This strategy is applied in the last test case, resulting in an improved availability (Fig. 8d). The results of the semi-Markov process are still under the assumption of perfect maintenance. Table II presents the running time for two problem instances of all four test cases, executed on a machine with two Intel Xeon CPU E5-2630 2.4 GHz processors and 64 GB memory. Both problem instances consider a time horizon of $10000 \mathrm{~h}$. In order to allow for fair comparison between the methods, both use the same numerical step-size $\mathrm{d} t$ and numerical methods with a global error of $\mathcal{O}\left(\mathrm{d} t^{2}\right)$. The first problem instance uses a step-size of $10 \mathrm{~h}$, where the second considers a step-size of $2 \mathrm{~h}$. Comparing test cases $\mathrm{S} / \mathrm{E} / \mathrm{R}$ and $\mathrm{M} / \mathrm{E} / \mathrm{R}$ shows the effect on the running time of increasing the problem size from $|\mathcal{S}|=4 /|\mathcal{F}|=1$ to $|\mathcal{S}|=10 /|\mathcal{F}|=3$. Furthermore, comparing test cases M/E/R and $\mathrm{M} / \mathrm{G} / \mathrm{R}$ shows the effect on the running time of transitions with a less thick-tailed pdf.

\section{CONCLUSION}

The paper presents a novel non-renewal stochastic process for the availability assessment of single-feeder IES sections, accurately taking into account competing risks, general transition rates and both minimal and perfect corrective maintenance. Additionally, the paper presents a solution strategy, which allows decoupling the time constants. The numerical illustration validates the proposed approach against the Markov and semi-Markov processes when satisfying respectively the Markov and renewal property. Directions for future work include extending the approach for multi-feeder sections and time-inhomogeneous transition rates. Additionally, the goal is

TABLE II

RUNNING TIMES FOR THE SEMI-MARKOV AND PRESENTED METHOD.

\begin{tabular}{ccrcr} 
& \multicolumn{2}{c}{ Semi-Markov method } & \multicolumn{2}{c}{ Presented method } \\
& $(10000 / 10)$ & $(10000 / 2)$ & $(10000 / 10)$ & $(10000 / 2)$ \\
\hline $\mathrm{S} / \mathrm{E} / \mathrm{R}$ & $2.71 \mathrm{sec}$ & $95.9 \mathrm{sec}$ & $0.68 \mathrm{sec}$ & $54.9 \mathrm{sec}$ \\
$\mathrm{M} / \mathrm{E} / \mathrm{R}$ & $7.24 \mathrm{sec}$ & $328.7 \mathrm{sec}$ & $2.83 \mathrm{sec}$ & $152.9 \mathrm{sec}$ \\
$\mathrm{M} / \mathrm{G} / \mathrm{R}$ & $2.44 \mathrm{sec}$ & $114.2 \mathrm{sec}$ & $0.74 \mathrm{sec}$ & $96.4 \mathrm{sec}$ \\
$\mathrm{M} / \mathrm{G} / \mathrm{B}$ & - & - & $1.30 \mathrm{sec}$ & $89.4 \mathrm{sec}$
\end{tabular}


to develop a combinatorial method that uses the presented approach to evaluate the availability of an IES combining several sections.

\section{ACKNOWLEDGMENT}

The authors would like to acknowledge the following people for their valuable contributions to the paper: Dr. H. Ergun, Dr. F. Geth and Prof. Dr. G. Deconinck.

\section{REFERENCES}

[1] International Electrotechnical Commission (IEC), "Availability, <of an item $>$," 2015. [Online]. Available: http://www.electropedia.org/iev/iev.nsf/display?openform\&ievref=192-01[Accessed: Dec. 4, 2017]

[2] M. de Nooij, C. Koopmans, and C. Bijvoet, "The value of supply security: the costs of power interruptions: economic input for damage reduction and investment in networks," Energy Econ., vol. 29, no. 2, pp. 277-295, 2007.

[3] G. H. Kjølle, K. Samdal, B. Singh, and O. A. Kvitastein, "Customer costs related to interruptions and voltage problems: methodology and results," IEEE Trans. Power Syst., vol. 23, no. 3, pp. 1030-1038, 2008.

[4] J. Y. Chan and J. V. Milanović, "Assessment of the economic value of voltage sag mitigation devices to sensitive industrial plants," IEEE Trans. Power Deliv., vol. 30, no. 6, pp. 2374-2382, 2015.

[5] H. Øye, "Power failure, temporary pot shut-down, restart and repair," in 27th Int. Alum. Conf., Moscow, 2012, pp. 12-14.

[6] International Electrotechnical Commission (IEC), "Reliability, <of an item >," 2015. [Online]. Available: http://www.electropedia.org/iev/iev.nsf/display?openform\&ievref=192-01-2 [Accessed: Dec. 4, 2017]

[7] - "Maintainability, $<$ of an item $>, " 2015$. [Online]. Available: http://www.electropedia.org/iev/iev.nsf/display?openform\&ievref=192-01-27 [Accessed: Dec. 4, 2017]

[8] H. Wang, "A survey of maintenance policies of deteriorating systems," Eur. J. Oper. Res., vol. 139, no. 3, pp. 469-489, 2002.

[9] International Electrotechnical Commission (IEC), "Recoverability, $<$ of an item $>$," 2015. [Online]. Available: http://www.electropedia.org/iev/iev.nsf/display?openform\&ievref=192-01[Accessed: Dec. 4, 2017]

[10] R. A. Howard, Dynamic Probabilistic Systems: Volume I, 2nd ed. Mineola, New York: Dover Publications, Inc., 1971.

[11] X. Bao and L. Cui, "An analysis of availability for series Markov repairable system with neglected or delayed failures," IEEE Trans. Reliab., vol. 59, no. 4, pp. 734-743, 2010.

[12] R. Moghaddass, M. J. Zuo, and J. Qu, "Reliability and availability analysis of a repairable k-out-of-n: G system with $\mathrm{R}$ repairmen subject to shut-off rules," IEEE Trans. Reliab., vol. 60, no. 3, pp. 658-666, 2011.

[13] S. Du, E. Zio, and R. Kang, "A new analytical approach for interval availability analysis of Markov repairable systems," IEEE Trans. Reliab., vol. 67 , no. 1, pp. 118-128, 2018.

[14] J. B. Bowles, "Commentary - caution: constant failure-rate models may be hazardous to your design," IEEE Trans. Reliab., vol. 51, no. 3, pp. 375-377, 2002.

[15] J. H. Jürgensen, L. Nordström, and P. Hilber, "A review and discussion of failure rate heterogeneity in power system reliability assessment," in 2016 Int. Conf. Probabilistic Methods Appl. to Power Syst., Beijing, 2016, pp. 1-8.

[16] M. Edimu, C. T. Gaunt, and R. Herman, "Using probability distribution functions in reliability analyses," Electr. Power Syst. Res., vol. 81, no. 4, pp. 915-921, 2011.
[17] R. A. Howard, Dynamic Probabilistic Systems: Volume II, 2nd ed. Mineola, New York: Dover Publications, Inc., 1971.

[18] G. Becker, L. Camarinopoulos, and G. Zioutas, "A semi-Markovian model allowing for inhomogenities with respect to process time," Reliab. Eng. Syst. Saf., vol. 70, no. 1, pp. 41-48, 2000.

[19] O. Chryssaphinou, N. Limnios, and S. Malefaki, "Multi-state reliability systems under discrete time semi-Markovian hypothesis," IEEE Trans. Reliab., vol. 60, no. 1, pp. 80-87, 2011.

[20] H. Dui, S. Si, M. J. Zuo, and S. Sun, "Semi-Markov process-based integrated importance measure for multi-state systems," IEEE Trans. Reliab., vol. 64, no. 2, pp. 754-765, 2015.

[21] R. G. Gallager, Stochastic Processes Theory for Application, 1st ed. Padstow Cornwall: Cambridge University Press, 2013.

[22] J. P. Kharoufeh, C. J. Solo, and M. Y. Ulukus, "Semi-Markov models for degradation-based reliability," IIE Trans., vol. 42, no. 8, pp. 599-612, 2010.

[23] International Electrotechnical Commission (IEC), "Radial system," $1985 . \quad$ [Online]. Available: http://www.electropedia.org/iev/iev.nsf/display?openform\&ievref=601-02-15 [Accessed: Dec. 4, 2017]

[24] N. Hritonenko and Y. Yatsenko, "Age-structured PDEs in economics, ecology, and demography: optimal control and sustainability," Math. Popul. Stud., vol. 17, no. 4, pp. 191-214, 2010

[25] S. I. Solodushkin, I. F. Yumanova, and R. H. De Staelen, "First order partial differential equations with time delay and retardation of a state variable," J. Comput. Appl. Math., vol. 289, pp. 322-330, 2015.

[26] O. Angulo, J. C. López-marcos, M. A. López-marcos, and F. A. Milner, "A numerical method for nonlinear age-structured population models with finite maximum age," J. Math. Anal. Appl., vol. 361, no. 1, pp. $150-160,2010$

[27] E. Hairer, S. Norsett, and G. Wanner, Solving Ordinary Differential Equations I, 1st ed. New York: Springer Berlin Heidelberg, 1993, vol. 8 .

[28] C. Moura and E. L. Droguett, "Numerical approach for assessing system dynamic availability via continuous time homogeneous semi-Markov processes," Methodol. Comput. Appl. Probab., vol. 12, no. 3, pp. 431$449,2010$.

Tom Van Acker (S'14) was born in 1990, in Roeselare, Belgium. He received the M.Eng. from the Katholieke Hogeschool Sint-Lieven, Ghent, Belgium in 2012 and the M.Sc. degrees in electrical engineering for the University of Leuven, Leuven, Belgium in 2014. Currently, he is working towards a $\mathrm{Ph} . \mathrm{D}$. degree in engineering at the Department of Electrical Engineering of the University of Leuven. His research is funded by a Ph.D. Fellowship of the Flemish Agency for Innovation \& Entrepreneurship in cooperation with BASF Antwerp NV (Grant No. 150257). His main area of research interest are industrial energy system availability and risk assessment, focussing on improved modeling of its stochastic behavior.

Dirk Van Hertem (S'02-SM'09) graduated as a M.Eng. in 2001 from the $\mathrm{KHK}$, Geel, Belgium and as a M.Sc. in Electrical Engineering from the KU Leuven, Belgium in 2003. In 2009, he has obtained his $\mathrm{PhD}$, also from the KU Leuven. In 2010, Dirk Van Hertem was a member of EPS group at the Royal Institute of Technology (KTH), in Stockholm. Since spring 2011 he is back at the University of Leuven where he is an associate professor in the ELECTA group. His special fields of interest are power system operation and control in systems with FACTS and HVDC and building the transmission system of the future, including offshore grids and the supergrid concept.

Dr. Van Hertem is an active member of both IEEE (PES and IAS) and Cigré, currently acting as chair of the IEEE Benelux section and as chair of the steering committee of the ISGT Europe conference. He was the general chair of the IEEE EnergyCon 2016 conference, held in Leuven. 